\title{
The Fast Simulation of Scattering Characteristics from a Simplified Time Varying Sea Surface
}

\author{
Yiwen Wei, ${ }^{1}$ Lixin Guo, ${ }^{1,2}$ and Xiao Meng' \\ ${ }^{1}$ School of Physics and Optoelectronic Engineering, Xidian University, Xian 710071, China \\ ${ }^{2}$ State Key Laboratory of Integrated Services Networks, Xidian University, Xi'an 710071, China \\ Correspondence should be addressed to Yiwen Wei; ywwei0910@gmail.com
}

Received 29 December 2014; Revised 2 April 2015; Accepted 7 April 2015

Academic Editor: Claudio Curcio

Copyright (c) 2015 Yiwen Wei et al. This is an open access article distributed under the Creative Commons Attribution License, which permits unrestricted use, distribution, and reproduction in any medium, provided the original work is properly cited.

\begin{abstract}
This paper aims at applying a simplified sea surface model into the physical optics (PO) method to accelerate the scattering calculation from 1D time varying sea surface. To reduce the number of the segments and make further improvement on the efficiency of PO method, a simplified sea surface is proposed. In this simplified sea surface, the geometry of long waves is locally approximated by tilted facets that are much longer than the electromagnetic wavelength. The capillary waves are considered to be sinusoidal line superimposing on the long waves. The wavenumber of the sinusoidal waves is supposed to satisfy the resonant condition of Bragg waves which is dominant in all the scattered short wave components. Since the capillary wave is periodical within one facet, an analytical integration of the PO term can be performed. The backscattering coefficient obtained from a simplified sea surface model agrees well with that obtained from a realistic sea surface. The Doppler shifts and width also agree well with the realistic model since the capillary waves are taken into consideration. The good agreements indicate that the simplified model is reasonable and valid in predicting both the scattering coefficients and the Doppler spectra.
\end{abstract}

\section{Introduction}

The calculation of electromagnetic (EM) scattering from a time varying surface is important in many fields such as radar surveillance, target tracking, and ocean remote sensing [1]. Useful techniques have already been developed to provide realistic results. They can be based on exact numerical methods (MoM, FEM, FDTD, and so on [2-5]) or approximate approaches [6]. Because numerical methods are unfortunately not efficient, the approximate approaches are widely used for the moment to calculate the scattered field from a large time varying surface. Among them, $\mathrm{PO}[7,8]$ is most employed because it is simple and easy to implement.

However, the PO method is still limited by the number of unknowns when dealing with large time varying sea surface. When dealing with the scattering problem form the sea, it should be noted that the sea surface should be divided into small segments whose length of each segment on the realistic sea surface should be 1/8 1/10 wavelength of the incident wave to accurately reflect the geometry characteristics of the sea. Since each segment is that small, the number of the segments form the realistic sea surface will be very large. To reduce the number of segments, one can divide the sea surface into larger segments. However, the larger the segment is, the more inaccuracy will be shown in the scattering coefficient and Doppler spectrum. The inaccurate results may be caused by two reasons. Firstly, the phase difference on one segment will be neglected in this case. This will lead to the inaccuracy of the integration on each segment. Secondly, the capillary waves superimposed on each segment are not taken into consideration. This will cause the fact that the Doppler value in some spectrum regions cannot be detected by the radar. Since the segment cannot be larger than 1/8 1/10 wavelength, the number of the unknowns will be tremendous especially when dealing with time varying large sea surface, which will limit the efficiency of PO method.

In order to get accurate results with larger segments, as well as promote the efficiency of conventional PO method, we add capillary waves [9]. The capillary wave considered to be sinusoidal line superimposing on each segment. The wavenumbers of the sinusoidal waves are supposed to satisfy 


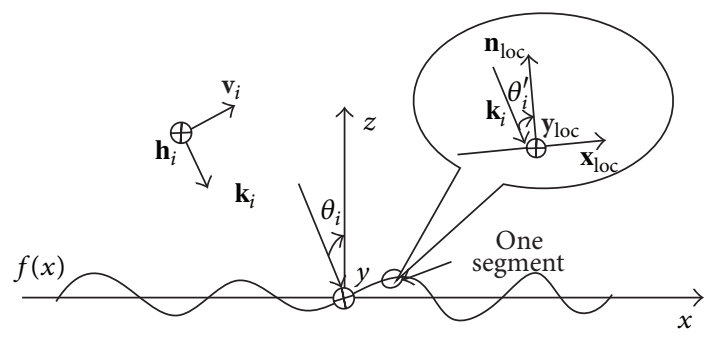

(a)

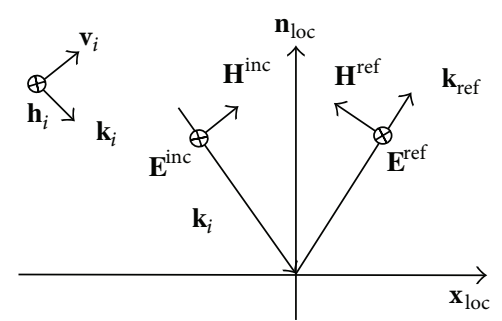

(b)

FIGURE 1: The illustration of the scattering problem from a sea surface, (a) the global coordinate system, and (b) the local coordinate system.

the resonant condition of Bragg waves which are predominant in the scattered short wave component. In this way, we substitute the simplified sea surface for the realistic sea surface approximately. Since the capillary wave is periodical within one facet, the analytical expression of the induced currents can be given after we get the currents on the first period of the sinusoidal wave.

This paper is organized as follows. In Section 2, the PO method is introduced and applied on the simplified sea surface model. All the formulas are derived and all the expressions are given in this part; both horizontal $(\mathrm{HH})$ polarization and vertical (VV) polarizations are considered. Several numerical simulations are exhibited in Section 3 to show the validity and efficiency of the new model compared with the realistic sea surface. Then, this model is used to investigate the characteristic of the Doppler spectrum of time varying sea surface. Section 4 ends with a summary of the new model and a proposition for further pertinent investigation.

\section{Formula}

2.1. The Physical Optics Formulation. The initial point of physical optics is the surface currents produced by an incoming electromagnetic wave $\left(\mathbf{E}^{\text {inc }}, \mathbf{H}^{\text {inc }}\right)$. Considering a $1 D$ sea surface, the induced electric currents $\mathbf{J}$ and magnetic currents $\mathbf{M}$ on each segment (the length of each segment is set as $1 / 8 \sim 1 / 10$ wavelength of the incident wave to meet the division criterion) are given by

$$
\mathbf{J}=\widehat{\mathbf{n}} \times \mathbf{H M}=\mathbf{E} \times \widehat{\mathbf{n}},
$$

where $\widehat{\mathbf{n}}$ is the unit normal vector of the surface and $\mathbf{r}$ indicates the position of each segment. $\mathbf{E}$ and $\mathbf{H}$ are, respectively, the total electric and magnetic fields at the surface. For TE case, the horizontal polarization vector of the incident wave $\widehat{\mathbf{h}}_{i}$ is along $\widehat{\mathbf{y}}, \widehat{\mathbf{k}}_{i}$ is the wavenumber of the incident wave, and the vertical polarization vector is $\widehat{\mathbf{v}}_{i}=\widehat{\mathbf{k}}_{i} \times \widehat{\mathbf{h}}_{i}$. The scattering problems in the global coordinate system and the local coordinate system are shown in Figures 1(a) and (b), respectively. Given the slop of one segment, the local coordinate system can be built as $\left\{\widehat{\mathbf{x}}_{\text {loc }}, \widehat{\mathbf{y}}_{\text {loc }}, \widehat{\mathbf{z}}_{\text {loc }}\right\}$, and each coordinate component can be expressed as

$$
\begin{aligned}
& \widehat{\mathbf{z}}_{\mathrm{loc}}=\widehat{\mathbf{n}}, \\
& \widehat{\mathbf{y}}_{\mathrm{loc}}=\widehat{\mathbf{y}}=\widehat{\mathbf{h}}_{i}, \\
& \widehat{\mathbf{x}}_{\mathrm{loc}}=\widehat{\mathbf{y}}_{\mathrm{loc}} \times \widehat{\mathbf{z}}_{\mathrm{loc}} .
\end{aligned}
$$

Let a plane wave illuminate on the rough sea surface; then the incident field on the surface can be given as

$$
\begin{aligned}
\mathbf{E}^{\text {inc }} & =\widehat{\mathbf{y}} E^{\text {inc }} \exp \left[i \overrightarrow{\mathbf{k}}_{i} \cdot \overrightarrow{\mathbf{r}}_{o}\right], \\
\mathbf{H}^{\text {inc }} & =\frac{\widehat{\mathbf{v}}_{i} E^{\text {inc }} \exp \left[i \overrightarrow{\mathbf{k}}_{i} \cdot \overrightarrow{\mathbf{r}}_{o}\right]}{\eta},
\end{aligned}
$$

where $\overrightarrow{\mathbf{r}}_{o}$ is the position of each surface segment and $\eta$ is the wave impedance in the free space.

Inserting the TE reflection coefficient $R_{\mathrm{TE}}$, the electric $\mathbf{E}^{\text {ref }}$ and magnetic $\mathbf{H}^{\text {ref }}$ reflected fields are

$$
\begin{aligned}
\mathbf{E}^{\mathrm{ref}} & =\widehat{\mathbf{h}}_{i} R_{\mathrm{TE}} E^{\mathrm{inc}} \exp \left[i \overrightarrow{\mathbf{k}}_{i} \cdot \overrightarrow{\mathbf{r}}_{o}\right] \\
\mathbf{H}^{\mathrm{ref}} & =\frac{\left(\widehat{\mathbf{k}}_{\mathrm{ref}} \times \widehat{\mathbf{h}}_{i}\right) E^{\mathrm{inc}} \exp \left[i \overrightarrow{\mathbf{k}}_{i} \cdot \overrightarrow{\mathbf{r}}_{o}\right]}{\eta},
\end{aligned}
$$

where $\widehat{\mathbf{k}}_{\text {ref }}=\widehat{\mathbf{k}}_{i}-2 \widehat{\mathbf{n}}\left(\widehat{\mathbf{k}}_{i} \cdot \widehat{\mathbf{n}}\right)$. Adding the contributions of incident and reflected field, the total electric and magnetic fields can be written as a function of the incident electric field, the reflection coefficients, and the intrinsic impedance of the first medium. Then, going back to the boundary 
conditions (1), the equivalent currents can be reckoned from the total fields. Consider

$$
\begin{aligned}
\mathbf{J}= & \widehat{\mathbf{n}} \times \mathbf{H}=\widehat{\mathbf{n}} \times\left(\mathbf{H}^{\mathrm{inc}}+\mathbf{H}^{\mathrm{ref}}\right) \\
= & \frac{E^{\mathrm{inc}} \exp \left[i \overrightarrow{\mathbf{k}}_{i} \cdot \overrightarrow{\mathbf{r}}_{o}\right]}{\eta} \\
& \cdot\left[\widehat{\mathbf{n}} \times \widehat{\mathbf{v}}_{i}+R_{\mathrm{TE}} \widehat{\mathbf{n}} \times\left(\widehat{\mathbf{k}}_{\mathrm{ref}} \times \widehat{\mathbf{h}}_{i}\right)\right] \\
= & \frac{E^{\mathrm{inc}} \exp \left[i \overrightarrow{\mathbf{k}}_{i} \cdot \overrightarrow{\mathbf{r}}_{o}\right]}{\eta} \cdot \cos \theta_{\mathrm{loc}}^{\mathrm{inc}} \cdot\left(1-R_{\mathrm{TE}}\right) \cdot \widehat{\mathbf{y}}_{\mathrm{loc}}, \\
\mathbf{M}= & \mathbf{E} \times \widehat{\mathbf{n}}=\left(\mathbf{E}^{\mathrm{inc}}+\mathbf{E}^{\mathrm{ref}}\right) \times \widehat{\mathbf{n}} \\
= & E^{\mathrm{inc}} \exp \left[i \overrightarrow{\mathbf{k}}_{i} \cdot \overrightarrow{\mathbf{r}}_{o}\right]\left(1+R_{\mathrm{TE}}\right) \cdot\left(\widehat{\mathbf{y}}_{\mathrm{loc}} \times \widehat{\mathbf{n}}\right) \\
= & E^{\mathrm{inc}} \exp \left[i \overrightarrow{\mathbf{k}}_{i} \cdot \overrightarrow{\mathbf{r}}_{o}\right]\left(1+R_{\mathrm{TE}}\right) \cdot \widehat{\mathbf{x}}_{\mathrm{loc}} \cdot
\end{aligned}
$$

The field scattered by the dielectric sea surface can readily be determined as the radiation of $\mathbf{J}$ and $\mathbf{M}$. The scattered field can be given by the well-known Stratton-Chu formula; that is,

$$
\begin{aligned}
E^{\mathrm{sca}} & =\mathbf{E}^{\mathrm{sca}} \cdot \widehat{\mathbf{h}}_{i} \\
& =i k \eta \int_{s} J G\left(\mathbf{r}, \mathbf{r}^{\prime}\right) d s^{\prime}+\int_{s} M\left(\widehat{\mathbf{n}}^{\prime} \cdot \nabla G\left(\mathbf{r}, \mathbf{r}^{\prime}\right)\right) d s^{\prime},
\end{aligned}
$$

where $\widehat{\mathbf{n}}^{\prime}$ is the unit normal vector of each segment. The resulting expressions are written in terms of Green's function $G\left(\mathbf{r}, \mathbf{r}^{\prime}\right)$ and $\widehat{\mathbf{n}}^{\prime} \cdot \nabla G\left(\mathbf{r}, \mathbf{r}^{\prime}\right)$. The far field approximation allows expressing these functions as [2]. Consider

$$
\begin{aligned}
& \left.G\left(\mathbf{r}, \mathbf{r}^{\prime}\right)\right|_{|\mathbf{r}| \rightarrow \infty} \\
& =\frac{i}{4} \sqrt{\frac{2}{\pi k_{0} r}} \exp \left(-i \frac{\pi}{4}\right) \\
& \cdot \exp \left(i k_{0} r\right) \exp \left[-i \overrightarrow{\mathbf{k}}_{s} \cdot \overrightarrow{\mathbf{r}}_{o}\right], \\
& \left.\widehat{\mathbf{n}}^{\prime} \cdot \nabla^{\prime} G\left(\mathbf{r}, \mathbf{r}^{\prime}\right)\right|_{|\mathbf{r}| \rightarrow \infty} \\
& =\frac{i}{4} \sqrt{\frac{2}{\pi k_{0} r} \exp \left(-i \frac{\pi}{4}\right)} \\
& \quad \cdot \exp \left(i k_{0} r\right)\left(-i \widehat{\mathbf{n}} \cdot \mathbf{k}_{s}\right) \exp \left[-i \overrightarrow{\mathbf{k}}_{s} \cdot \overrightarrow{\mathbf{r}}_{o}\right],
\end{aligned}
$$

where $\overrightarrow{\mathbf{k}}_{s}$ is the scattering wavenumber vector $\overrightarrow{\mathbf{k}}_{s}=$ $k_{0}\left(\sin \theta_{s} \widehat{\mathbf{x}}+\cos \theta_{s} \widehat{\mathbf{y}}\right)$. Consequently, the scattered fields are

$$
\begin{aligned}
E^{\mathrm{sca}}= & -\sqrt{\frac{k}{8 \pi r}} \exp \left(-i \frac{\pi}{4}\right) \exp \left(i k_{0} r\right) \\
& \cdot \int_{s} \exp \left(-i \overrightarrow{\mathbf{k}}_{s} \cdot \overrightarrow{\mathbf{r}}_{o}\right)\left[\eta \cdot J-M \cdot\left(\widehat{\mathbf{n}} \cdot \widehat{\mathbf{k}}_{s}\right)\right] d s^{\prime} .
\end{aligned}
$$

Similarly, when the sea surface is illuminated by the incident field $\mathbf{H}^{\text {inc }}=\widehat{\mathbf{y}} H^{\mathrm{inc}} \exp \left[i \overrightarrow{\mathbf{k}}_{i} \cdot \overrightarrow{\mathbf{r}}_{o}\right]$ (VV polarization), the induced electric and magnetic currents can be written as

$$
\begin{aligned}
\mathbf{J} & =H^{\mathrm{inc}} \exp \left[i \overrightarrow{\mathbf{k}}_{i} \cdot \overrightarrow{\mathbf{r}}_{o}\right] \cdot\left(1+R_{\mathrm{TM}}\right) \widehat{\mathbf{x}}_{\mathrm{loc}} \\
\mathbf{M} & =H^{\mathrm{inc}} \exp \left[i \overrightarrow{\mathbf{k}}_{i} \cdot \overrightarrow{\mathbf{r}}_{o}\right] \cdot\left(1-R_{\mathrm{TM}}\right) \cdot \cos \theta_{\mathrm{loc}}^{\mathrm{inc}} \cdot \widehat{\mathbf{y}}_{\mathrm{loc}}
\end{aligned}
$$

the scattered fields are

$$
\begin{aligned}
H^{\text {sca }}= & -\sqrt{\frac{k}{8 \pi r}} \exp \left(-i \frac{\pi}{4}\right) \exp (i k r) \\
& \cdot \int_{s} \exp \left(-i \overrightarrow{\mathbf{k}}_{s} \cdot \overrightarrow{\mathbf{r}}_{o}\right)\left[\eta \cdot J-M \cdot\left(\widehat{\mathbf{n}} \cdot \widehat{\mathbf{k}}_{s}\right)\right] d s^{\prime} .
\end{aligned}
$$

The analytical expression of scattering coefficient can be defined as

$$
\sigma=\lim _{r \rightarrow \infty} \frac{2 \pi r\left|E^{\mathrm{sca}}\right|^{2} /\left|E^{\mathrm{inc}}\right|^{2}}{L}
$$

wherein $L$ is the length of the sea surface.

The Doppler spectrum is a power spectral density of the random time varying complex amplitude of the sea surface scattering field; to evaluate it, we used a standard spectral estimation technique. Thus, the expression of the Doppler spectrum is defined as

$$
S(f)=\frac{1}{T}\left\langle\left|\int_{0}^{T} E^{\mathrm{sca}}(t) \exp (i 2 \pi f t) d t\right|^{2}\right\rangle
$$

where the angular bracket $\langle\cdot\rangle$ stands for the averaging over all the surface realizations. $T$ is the evolution time of the time varying sea surface.

2.2. The Simplified Sea Surface Model. In this simplified sea surface model, each segment on the sea surface is much longer than the wavelength of the incident wave. All segments are locally approximated by tilted facet. (In the following part of the paper, the "large segments" are all named as "facet.") These facets are centered on the grid points that are geometrically described by a discrete set of $f_{g}(x, t) . f_{g}(x, t)$ can be regarded as the gravity wave. The sinusoidal capillary waves are added on the planar facet. The surface profile within a planar facet can be represented as

$$
\begin{aligned}
f(x, t)= & f_{g}\left(x_{o}, t\right)+f_{g}^{\prime}\left(x-x_{o}\right)+B\left(k_{c}\right) \\
& \cdot \cos \left(k_{c} x+\omega_{c} t+\varphi\right),
\end{aligned}
$$

where $f$ is the height of the simplified sea surface, $B(\cdot)$ represents the amplitude of the superimposed capillary wave, and $k_{c}$ is the spatially short wavenumber, which is supposed to satisfy the resonant condition of Bragg waves. The definitions of $B\left(k_{c}\right)$ and $k_{c}$ will be discussed in the following in detail; $\omega_{c}$ represents the circle frequency, $\omega_{c}^{2}=g k_{c}^{3} / k_{m}^{3}$, and $g=$ $9.8 \mathrm{~m} / \mathrm{s}, k_{m} \approx 370 \mathrm{rad} / \mathrm{m} ; \varphi$ is the random phase of the ripple 


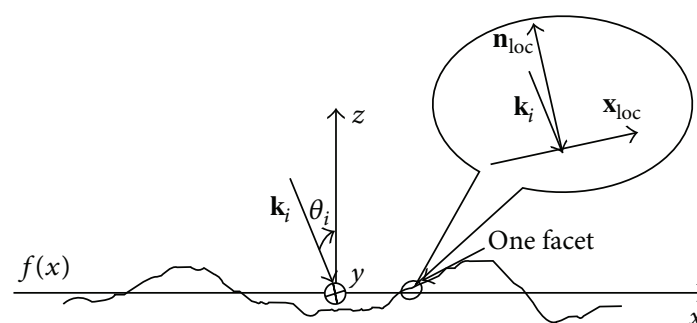

(a)

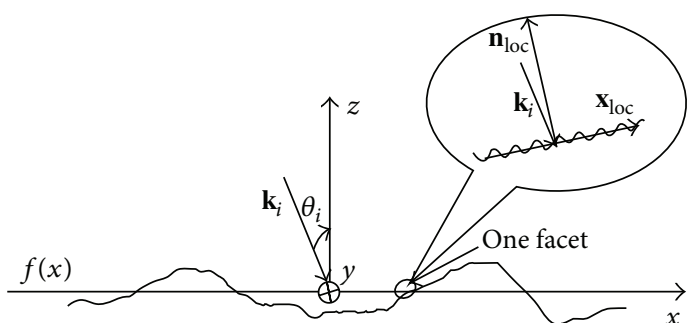

(b)

FIGURE 2: Configuration of sea surface; (a) sea profile constructed by large facet: simplified sea surface without capillary wave; (b) the simplified sea surface: capillary waves are added.

component. $f_{g}\left(x_{o}, t\right)$ is the height of the gravity wave in the middle point of the facet, and $f_{g}^{\prime}$ is the slope of the facet.

The configuration of the sea surface is depicted in Figure 2. Figure 2(a) shows the large-scale surface. Each facet is flat in this model. In Figure 2(b), the capillary waves are added and each facet is modified by sinusoidal waves.

Since the sinusoidal ripple is superimposed at microscopic level and is relevant for the Bragg resonant scattering mechanism, we assume that the echo waves are reflected by the wave crest of the resonant sinusoidal ripples. As Figure 3(a) shows, $\mathbf{q}=\mathbf{k}_{s}-\mathbf{k}_{i}$, and $\mathbf{q}_{l}$ is its projection on the plane facet. $\lambda_{l}$ is the wavelength of the resonant sinusoidal ripples on the plane facet. $\lambda_{c}$ is the corresponding wavelength on the $x$-axis. To meet the resonant condition, $\lambda_{l}$ is set as

$$
\lambda_{l}=\frac{\pi}{k \sin \theta_{i}^{\prime}}=\lambda_{c} \sqrt{1+\left(f_{g}^{\prime}\right)^{2}} .
$$

The wavenumber of the sinusoidal ripple is $k_{c}$, which can be written as $k_{c}=2 \pi / \lambda_{c}$. According to (15), $k_{c}$ can be determined as

$$
k_{c}=\frac{\pi}{k \sin \theta_{i}^{\prime} \sqrt{1+\left(f_{g}^{\prime}\right)^{2}}}
$$

and the amplitude of the ripple in (14) could be expressed by $B\left(k_{c}\right)=\pi \sqrt{2 S\left(k_{c}\right) / \Delta x_{g}} ; S\left(k_{c}\right)$ is the capillary spectrum located in the higher part of the sea spectrum, and $\Delta x_{g}$ is the length of the facet.

Figure 3(b) shows that the facet is divided into $N_{p}$ periods, $N_{p}=\operatorname{int}\left(\Delta x_{g} / \lambda_{c}\right)$. The currents in the first period can be expressed as (5), (6), and (10). Each period is divided into small segments. The lengths of the small segments are set as 1/8 1/10 wavelength of the incident wave to meet the division criterion. For the case of $\mathrm{HH}$ polarization, by moving the $\exp \left(-i \overrightarrow{\mathbf{k}}_{i} \cdot \overrightarrow{\mathbf{r}}_{o}\right)$ term from (5) and (6) to (8) and moving the $\widehat{\mathbf{n}} \cdot \widehat{\mathbf{k}}_{s}$ term from (8) to (5) and (6), $1 / \eta$ in (5) is removed, since it will counteract with the $\eta$ in (7). Then the currents can be rewritten as

$$
\begin{gathered}
J_{m n}=E^{\mathrm{inc}} \cos \theta_{\mathrm{loc}}^{\mathrm{inc}} \cdot\left(1-R_{\mathrm{TE}}\right) \cdot d l \\
M_{m n}=E^{\mathrm{inc}}\left(1+R_{\mathrm{TE}}\right) \cdot\left(\widehat{\mathbf{n}} \cdot \widehat{\mathbf{k}}_{s}\right) \cdot d l,
\end{gathered}
$$

where the subscripts $m$ and $n$ indicate the $m$ th segment in one period and the $n$th period on one facet, respectively. Consider $d l=\Delta x_{c} \sqrt{1+\left(f_{c}^{\prime}\right)^{2}} \cdot f_{c}^{\prime}$, which is the slope of each segment, is periodical on one facet. $f_{c}^{\prime}$ is the differential of $f(x, t)$. On each facet, $f_{c}^{\prime}$ can be expressed as

$$
f_{c}^{\prime}=f^{\prime}(x, t)=f_{g}^{\prime}-B\left(k_{c}\right) k_{c} \sin \left(k_{c} x+\omega_{c} t\right) ;
$$

the scattered field from one facet can be expressed as

$$
E^{\mathrm{sca}}=\text { coef } \cdot \sum_{m=1}^{N_{s}} \sum_{n=1}^{N_{p}} \exp \left(-i \overrightarrow{\mathbf{q}} \cdot \overrightarrow{\mathbf{r}}_{m n}\right)\left(J_{m n}-M_{m n}\right)
$$

where $N_{s}$ is the number of the segments in one period, $N_{s}=$ $\operatorname{int}\left(\lambda_{c} / \Delta x_{c}\right)$, and

$$
\text { coef }=-\sqrt{\frac{k}{8 \pi r}} \exp \left(-i \frac{\pi}{4}\right) \exp \left(i k_{0} r\right) .
$$

Since $J_{m n}$ and $M_{m n}$ vary periodically on one facet, (19) can be rewritten as

$$
\begin{aligned}
E^{\mathrm{sca}} & =\text { coef } \\
& \cdot \sum_{m=1}^{N_{s}}\left\{\sum_{n=1}^{N_{p}}\left(J_{m n}-M_{m n}\right) \exp \left(-i \overrightarrow{\mathbf{q}} \cdot \overrightarrow{\mathbf{r}}_{m n}\right)\right\}=\text { coef } \\
& \cdot \sum_{m=1}^{N_{s}}\left[\left(J_{m 1}-M_{m 1}\right) \sum_{n=1}^{N_{p}} \exp \left(-i \overrightarrow{\mathbf{q}} \cdot \overrightarrow{\mathbf{r}}_{m n}\right)\right] \\
& =\operatorname{coef} \sum_{m=1}^{N_{s}}\left[\exp \left(-i \overrightarrow{\mathbf{q}} \cdot \overrightarrow{\mathbf{r}}_{m 1}\right)\left(J_{m 1}-M_{m 1}\right)\right. \\
& \left.\cdot \sum_{n=1}^{N_{p}} \exp [-i \overrightarrow{\mathbf{q}}(n-1) \Delta \overrightarrow{\mathbf{r}}]\right]
\end{aligned}
$$




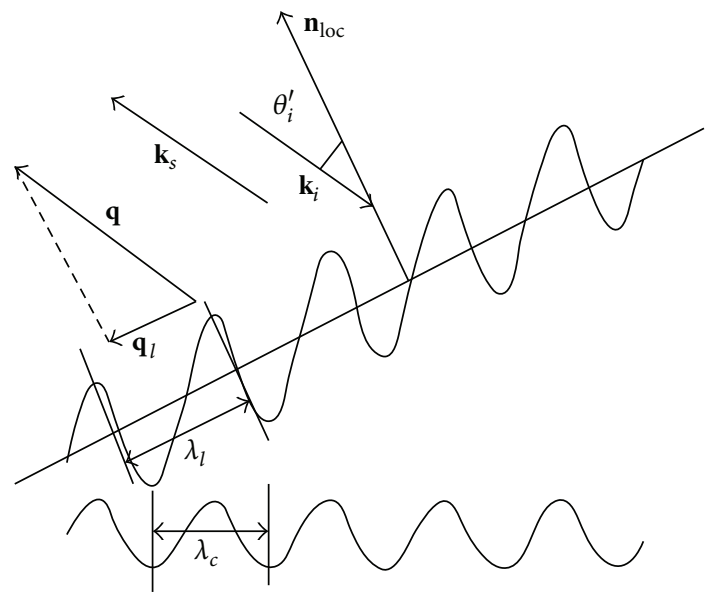

(a)

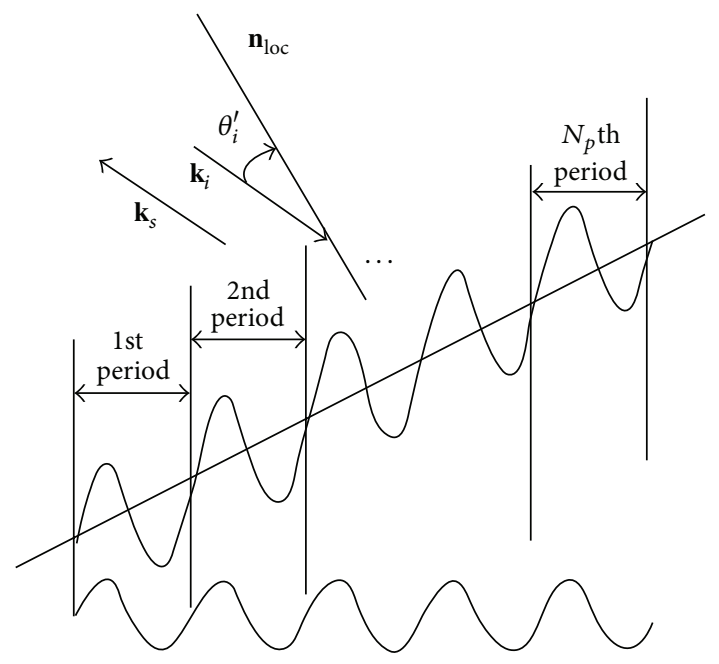

(b)

FIgURE 3: The ripples on one facet.

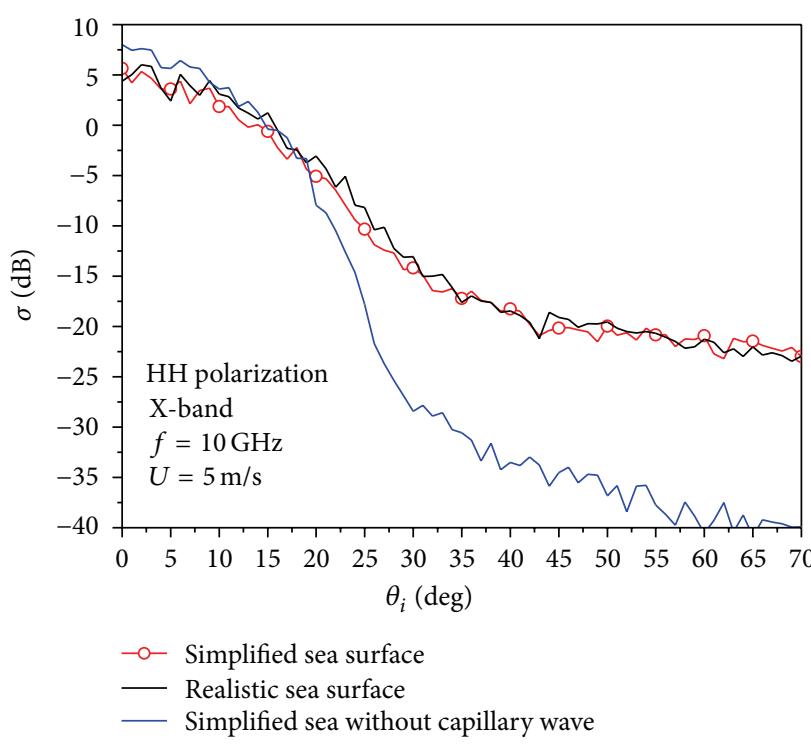

(a)

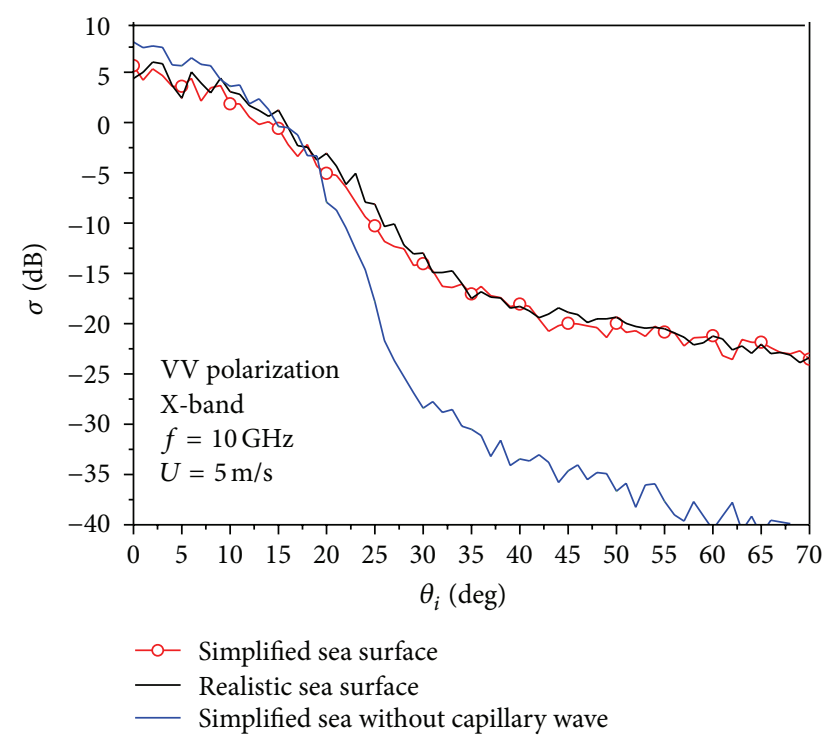

(b)

FIGURE 4: Backscattering coefficient from sea surface. (a) HH and (b) VV.

where $\Delta \overrightarrow{\mathbf{r}}$ is fixed on one facet, and it can be expressed as $\Delta \overrightarrow{\mathbf{r}}=\lambda_{c} \widehat{\mathbf{x}}+\lambda_{c} f_{g}^{\prime} \widehat{\mathbf{z}}$. Thus the scattered field from one facet can be obtained by

$$
\begin{aligned}
E^{\mathrm{sca}}= & \operatorname{coef} \sum_{m=1}^{N_{s}} \exp \left(-i \overrightarrow{\mathbf{q}} \cdot \overrightarrow{\mathbf{r}}_{m 1}\right)\left(J_{m 1}-M_{m 1}\right) \\
& \cdot \exp \left[-i\left(N_{p}-1\right) \cdot \frac{\overrightarrow{\mathbf{q}} \cdot \Delta \overrightarrow{\mathbf{r}}}{2}\right] \\
& \cdot \frac{\sin \left(\overrightarrow{\mathbf{q}} \cdot \Delta \overrightarrow{\mathbf{r}} \cdot N_{p} / 2\right)}{\sin (\overrightarrow{\mathbf{q}} \cdot \Delta \overrightarrow{\mathbf{r}} / 2)} .
\end{aligned}
$$

Summarizing the scattered field from all the facets, we can obtain the total field from the sea surface.

\section{Numerical Simulations and Discussions}

To validate the proposed simplified sea surface model, simulation results obtained from the simplified sea surface, the realistic sea surface, and the simplified sea without capillary wave will be shown and compared in the following.

Figure 4 shows the comparison of the presented simplified model with the realistic sea surface for the angular distribution of the backscattering coefficient (BSC). The geometry of observation is monostatic. Both $\mathrm{HH}$ (a) and VV (b) polarization are taken into consideration. The results are 


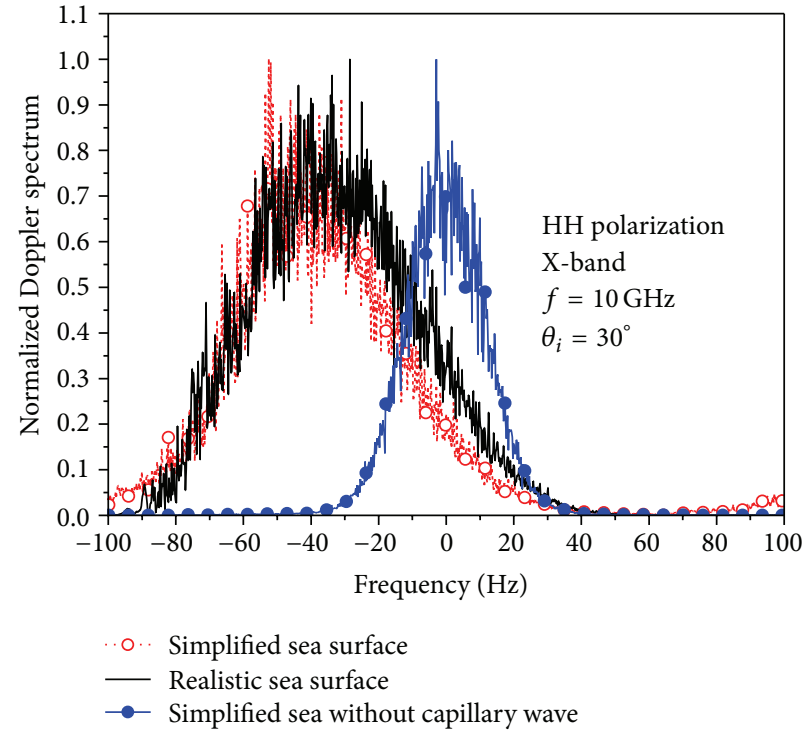

(a)

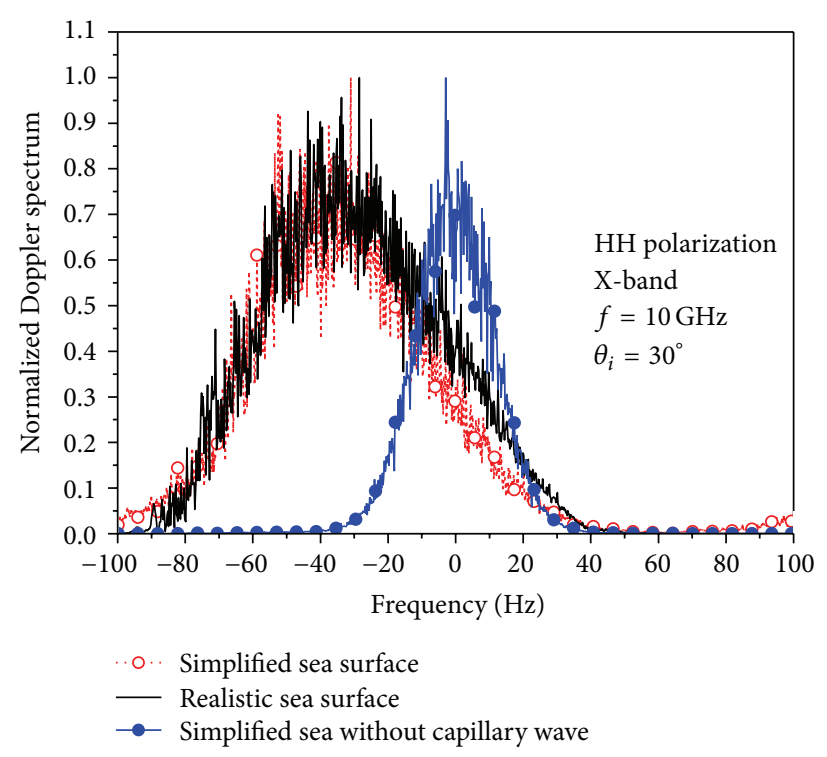

(b)

FIGURE 5: Doppler spectra from time varying sea surface, (a) HH and (b) VV.

obtained by averaging 50 surface realizations. The surfaces are generated using Monte Carlo [2] methods with 50 sets of Gaussian random numbers whose mean value is 0 and variance is 1 . The random numbers are called using the IMSL function library with 50 different seeds. The parameters in the simulation are as follows: the frequency of the incident wave is at the X-band $(10 \mathrm{GHz})$, the incident angle varies from $0^{\circ}$ to $70^{\circ}$, the length of the sea surface is $245.76 \mathrm{~m}$, the length of each facet is set to $1.92 \mathrm{~m}$, the wind speed at $10 \mathrm{~m}$ above the mean sea level is $U_{10}=5 \mathrm{~m} / \mathrm{s}$, and the relative permittivity of the sea water is calculated as $\varepsilon=(56.18,34.42)$ according to the Klein model [10] at $20^{\circ} \mathrm{C}$ and $32.5 \%$ of salinity. It is obvious that the BSC obtained from the proposed sea surface model and the realistic model are in fairly good agreement for the whole backscattering region for both polarizations. The BSC obtained by a simplified sea surface without capillary wave have an obvious discrepancy with the other two curves. Thus, the presented model is reliable for predicting the backscattering coefficient. We can also conclude that the resonant capillary wave plays an important role in the scattering result. The simulation time obtained from the simplified sea surface and realistic sea surface is also presented in Table 1 . The superiority of the simplified sea surface in terms of computation time is obvious. All results are obtained on a computer with a $2.93 \mathrm{GHz}$ processor (Intel Core i3 CPU), 3.45 GB memory, and Visual Fortran 6.5 compiler. All the comparisons of scattering results and the simulating time indicate that the proposed sea surface model is an accurate and efficient model.

Subsequently, the Doppler behaviors are observed with incident angle $\theta_{i}=30^{\circ}$ and frequency $f=10.0 \mathrm{GHz}$. The results obtained from the proposed simplified sea surface are compared with the result from the realistic sea surface and from the simplified sea without capillary wave (Figure 5). These results are averaged over 50 samples and normalized
TABLE 1: Comparison of simulating time by two methods.

\begin{tabular}{lccc}
\hline \multicolumn{2}{c}{ Backscattering coefficient } & \multicolumn{2}{c}{ Doppler spectrum } \\
Model & Time $(\mathrm{s})$ & Model & Time $(\mathrm{s})$ \\
\hline Simplified sea surface & 18.525 & Simplified sea surface & 478.938 \\
Realistic sea surface & 62.323 & Realistic sea surface & 1089.994 \\
\hline
\end{tabular}

by their respective maximum values. For the time-evolving simulations, the time step $\Delta t$ is selected as $0.005 \mathrm{~s}$, and 1024 steps are selected for the sea surface realization. Thus, the evolution time $T$ is $5.12 \mathrm{~s}$. The other parameters are the same as those given in Figure 4. The two curves obtained from the proposed sea surface model and the realistic model well agree for both polarizations. Doppler spectrum obtained from the simplified sea without capillary waves cannot reflect the Doppler characteristics of the sea surface. It should be noticed that the Doppler shifts are almost the same for both methods, whereas the Doppler width of the proposed method is a little smaller than the realistic sea surface. It is mainly caused by the fact that the other components of the capillary waves are supposed to be absent. After all, we conclude that the simplified sea surface model is accurate enough and convincible to describe the scattering characteristics of the sea.

The simulation times of Doppler spectrum of both sea surface models are presented in Table 1 . The simplified sea surface model takes much less time than the realistic sea surface, especially in the Doppler spectrum simulation.

In Figure 6, the Doppler spectra are illustrated for wind speed of $5 \mathrm{~m} / \mathrm{s}$ at different incident angles. The other parameters in calculation are the same as those given in Figure 5. With the incident angle increasing, the widths of the Doppler spectra first become broader and then shrink. Figure 7 shows the Doppler spectrum at different wind speed. The incident 


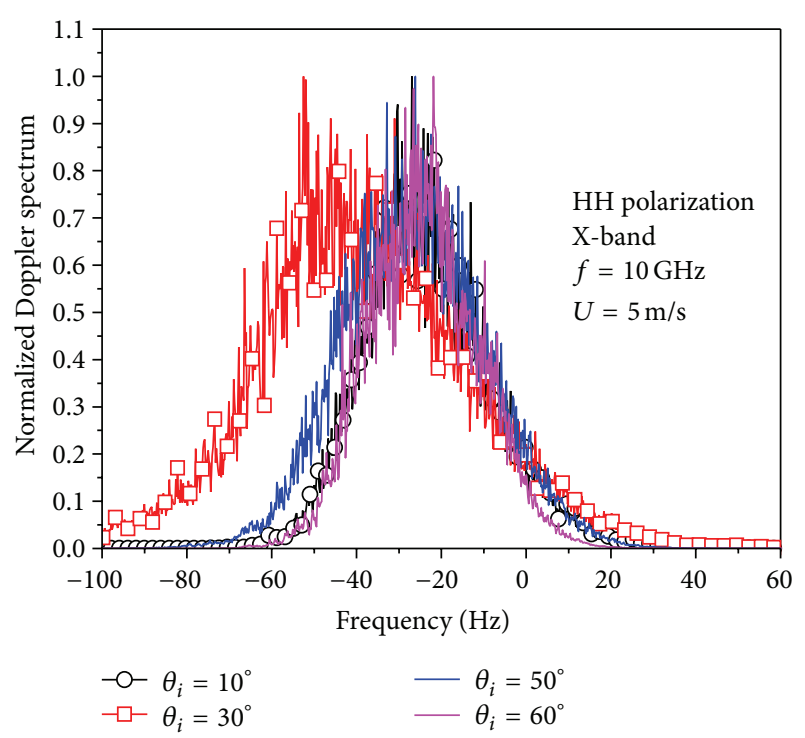

FIGURE 6: Doppler spectra of different incident angle.

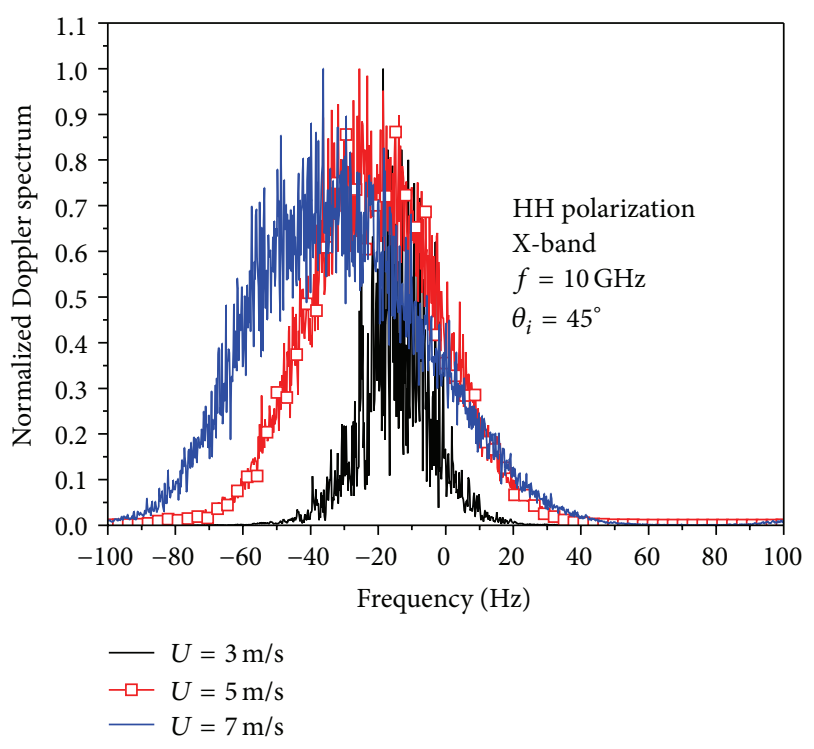

FIGURE 7: Doppler spectra at different wind speed.

angle is set as $\theta_{i}=45^{\circ}$. We can find that with the increasing wind speeds, the velocities of the surface water and the orbital motion for the large-scale sea wave increase, and there has been a gradual increase for the Doppler spectrum frequency shift, which is coincident with the measuring result by Rozenberg et al. [11]. On the other hand, the Doppler spectrum broadens with the wind speed increasing in the roughness of the sea surface. Thus the backscattering energy will be distributed over a wide region of the frequency domain, which has been discussed in $[12,13]$. All these results can demonstrate that the simplified sea surface model is valid to analyze the Doppler characteristic of the time varying sea surface.

\section{Conclusions}

The PO method is combined with the simplified sea surface model to calculate the backscattering coefficient and Doppler spectrum from time varying sea surface. With the simplified model, the efficiency of PO method is promoted. The characteristic of the Doppler spectrum of time varying sea surface is also investigated by the proposed model. Some shortcomings still exist in this method. Since the coupling between different segments are not taken into consideration, this method is limited to deal with the sea whose sea state is low or moderate. And this method will be inaccurate if the incident angle is large. The $2 \mathrm{D}$ problem is not considered in this paper. We will pay more attention to these remaining problems in the future study.

\section{Conflict of Interests}

The authors declare that there is no conflict of interests regarding the publication of this paper.

\section{Acknowledgments}

This work was supported by the National Natural Science Foundation for Distinguished Young Scholars of China (Grant no. 61225002), the Specialized Research Fund for the Doctoral Program of Higher Education (Grant no. 20100203110016), and the Fundamental Research Funds for the Central Universities (Grant no. K5051007001).

\section{References}

[1] F. T. Ulaby, R. K. Moore, and A. K. Fung, Microwave Remote Sesing (Active and Passive), Addison Wesley, New York, NY, USA, 1982.

[2] L. Tsang and J. A. Kong, Scattering of Electromagnetic Waves, vol. 2, John Wiley \& Sons, 2001.

[3] L. Kuang and Y. Q. Jin, "Bistatic scattering from a threedimensional object over a randomly rough surface using the FDTD algorithm," IEEE Transactions on Antennas and Propagation, vol. 55, no. 8, pp. 2302-2312, 2007.

[4] S. H. Lou, L. Tsang, and C. H. Chan, "Application of the finite element method to Monte Carlo simulations of scattering of waves by random rough surfaces: penetrable case," Waves in Random Media, vol. 1, no. 4, pp. 287-307, 1991.

[5] Y. W. Wei, L. X. Guo, A. Q. Wang, and Z. S. Wu, "Application of multiregion model to EM scattering from a dielectric rough surface with or without a target above it," IEEE Transactions on Antennas and Propagation, vol. 61, no. 11, pp. 5607-5620, 2013.

[6] T. M. Elfouhaily and C.-A. Guérin, "A critical survey of approximate scattering wave theories from random rough surfaces," Waves in Random Media, vol. 14, no. 4, pp. R1-R40, 2004.

[7] W. B. Gordon, "High frequency approximation to the physical optics scattering integral," IEEE Transactions on Antennas and Propagation, vol. 42, no. 3, pp. 427-432, 1994.

[8] J. T. Johnson, "On the geometrical optics and physical optics approximations for scattering from exponentially correlated surfaces," IEEE Transactions on Antennas and Propagation, vol. 45, pp. 2619-2629, 1994. 
[9] M. Zhang, H. Chen, and H.-C. Yin, "Facet-based investigation on em scattering from electrically large sea surface with twoscale profiles: theoretical model," IEEE Transactions on Geoscience and Remote Sensing, vol. 49, no. 6, pp. 1967-1975, 2011.

[10] L. A. Klein and C. T. Swift, "An improved model for the dielectric constant of sea water at microwave frequencies," IEEE Transactions on Antennas and Propagation, vol. AP-25, no. 1, pp. 104-111, 1977.

[11] A. D. Rozenberg, D. C. Quigley, and W. Kendall Melville, "Laboratory study of polarized scattering by surface waves at grazing incidence. I: wind waves," IEEE Transactions on Geoscience and Remote Sensing, vol. 33, no. 4, pp. 1037-1046, 1995.

[12] J. T. Johnson, J. V. Toporkov, and G. S. Brown, "A numerical study of backscattering from time-evolving sea surfaces: comparison of hydrodynamic models," IEEE Transactions on Geoscience and Remote Sensing, vol. 39, no. 11, pp. 2411-2420, 2001.

[13] J. V. Toporkov and G. S. Brown, "Numerical study of the extended Kirchhoff approach and the lowest order small slope approximation for scattering from ocean-like surfaces: doppler analysis," IEEE Transactions on Antennas and Propagation, vol. 50, no. 4, pp. 417-425, 2002. 

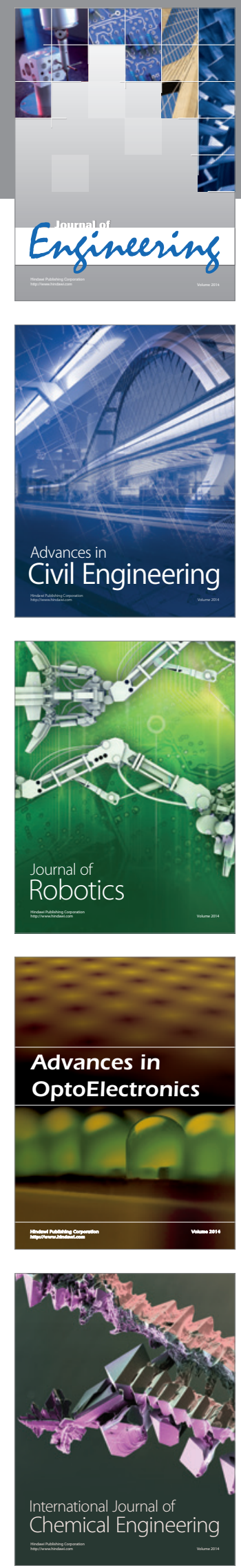

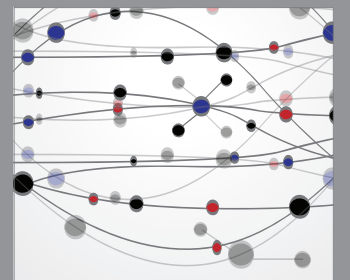

The Scientific World Journal
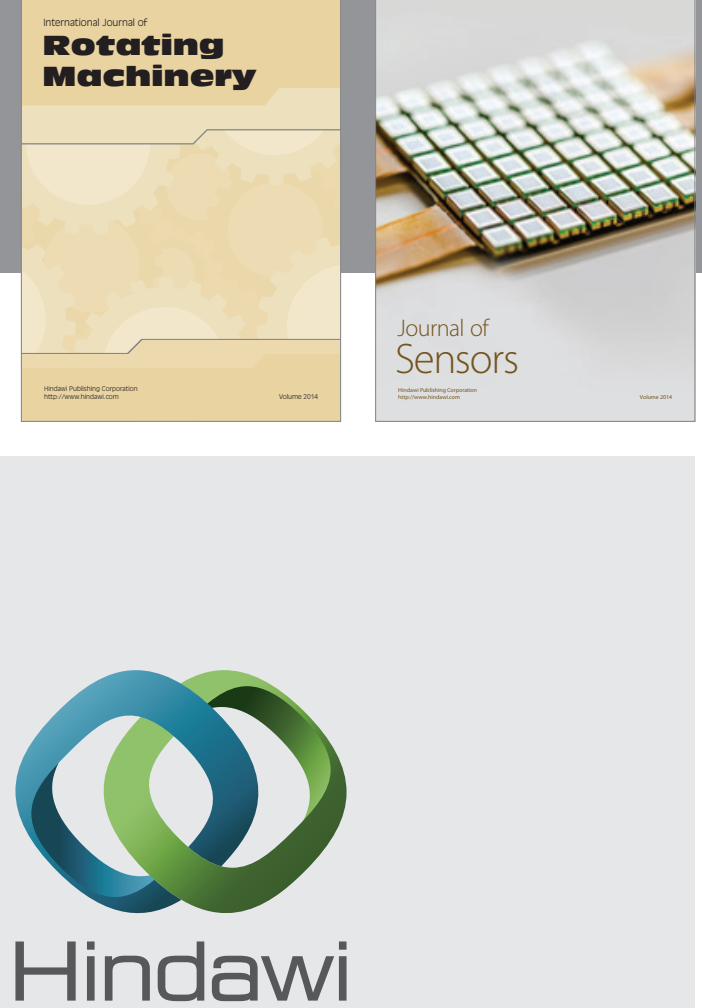

Submit your manuscripts at http://www.hindawi.com
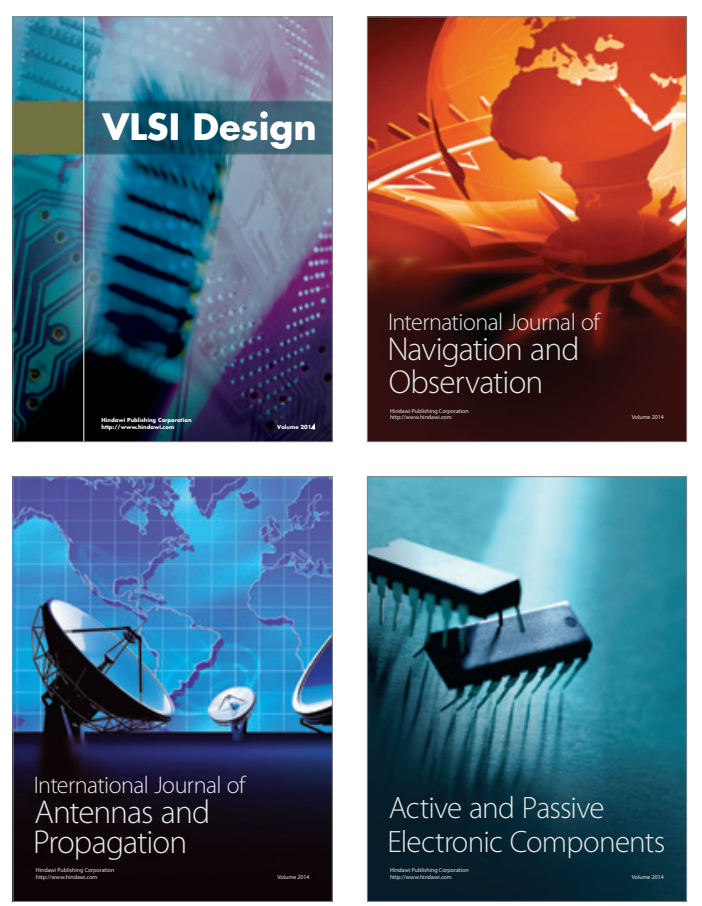
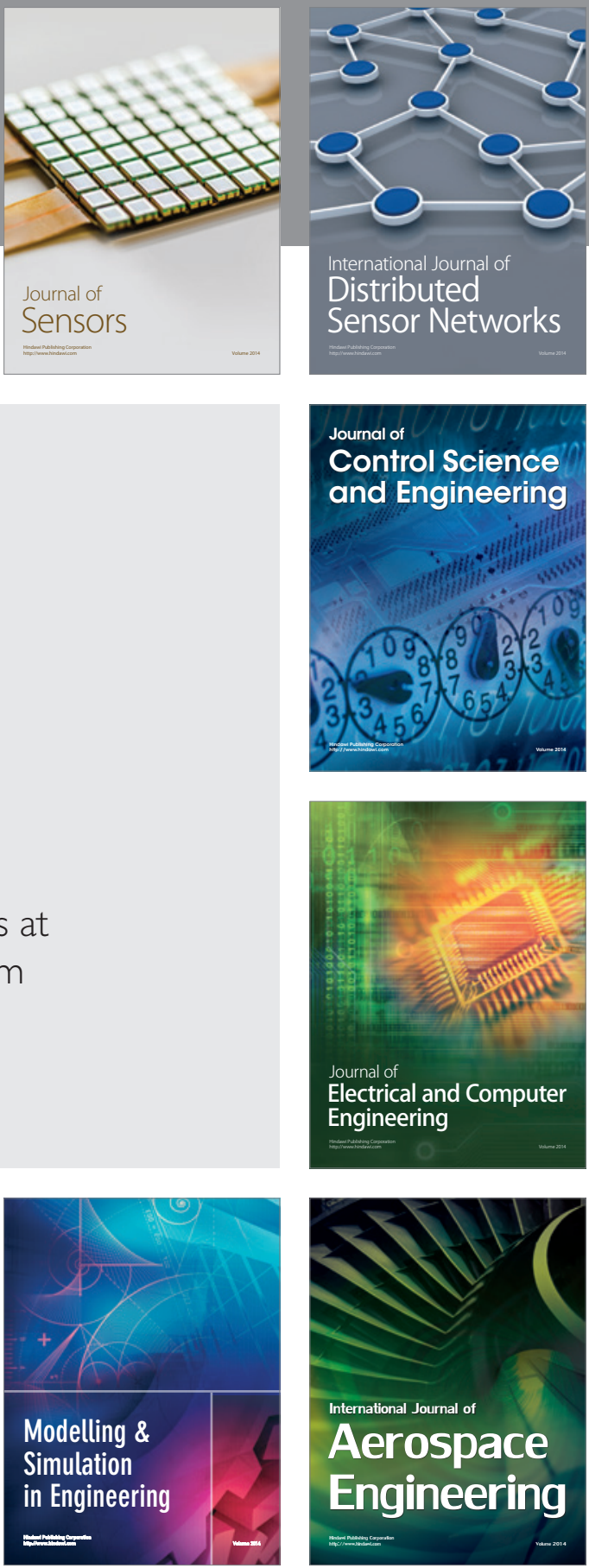

Journal of

Control Science

and Engineering
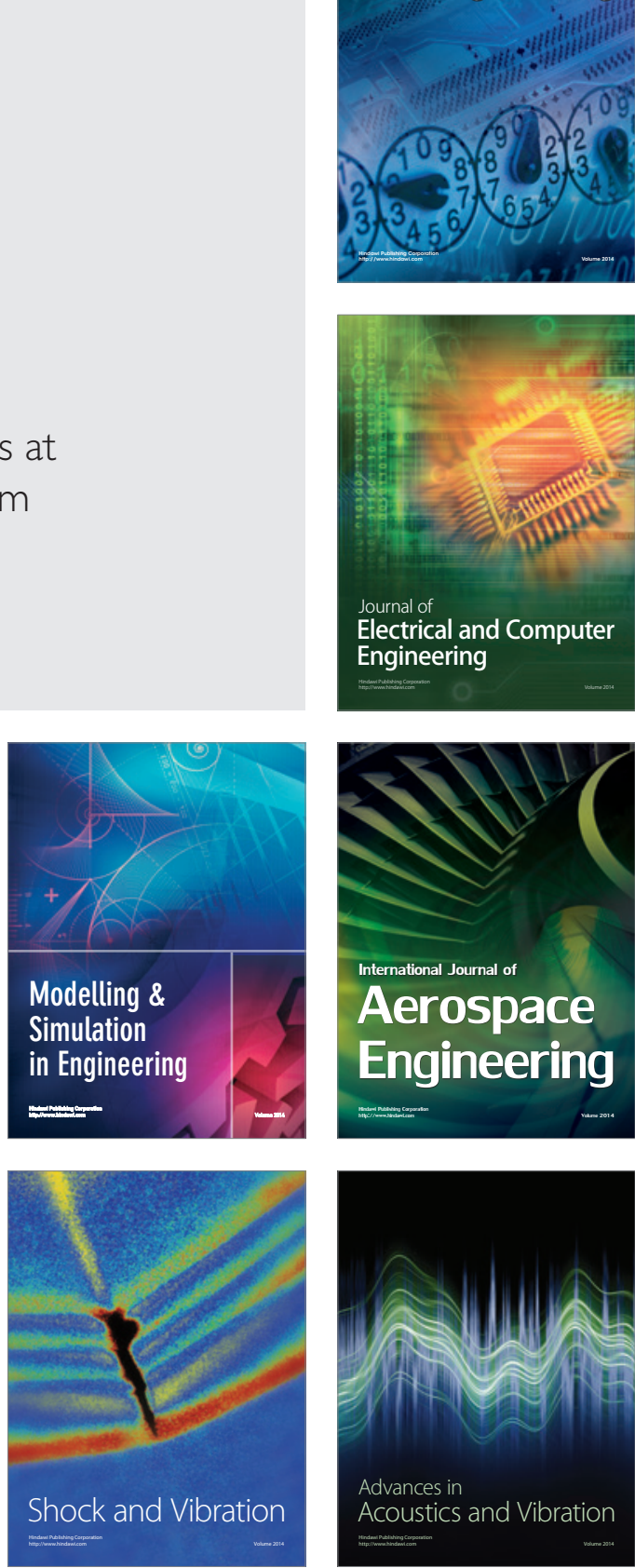